\title{
A Study on Discrete-Time Movement Models
}

\author{
Ionut Paun, Dirk Husmeier, Colin Torney \\ University of Glasgow \\ University of Glasgow, Glasgow, United Kingdom \\ i.paun.1@ research.gla.ac.uk; dirk.husmeier@glasgow.ac.uk \\ Colin.Torney@glasgow.ac.uk
}

\begin{abstract}
Understanding animal movement is an important challenge in ecology, with improvement in tagging technology permitting the collection of data on an increasingly wide range of species. Consequently, methodologies for statistical analysis of such data has received considerable attention in recent years. Discrete-time random walks are the foundation of the movement data models. The advantages of the discrete-time movement models are that they are intuitive and easily implemented, however the specification of the discretization step is often problematic and must be done in advance. Misspecification of the discretization step might lead to a model mismatch and thus choosing an appropriate test statistic to capture the model mismatch is essential.
\end{abstract}

Keywords: Discrete-time random walk, Time-step, Model checking, Diffusion coefficient

\section{Introduction}

Random walks are one of the most common and simple methods that are used to model movement data in a wide range of biological settings, such as cell movement [1] or animal movement [2]. There are different types of random walks, the simplest of them is the Brownian motion, where the movement is uncorrelated and unbiased. Uncorrelated means that the direction of the movement is not influenced by the previous directions moved. Unbiased means that there is no preference for a particular direction. One example of a very simple uncorrelated and unbiased random walk would be a random walk restricted on a lattice, where you have equal probability of going either up and down, or left or right. Also, Brownian motion can be shown to produce the heat equation (or standard diffusion). However, animal and cell movement is not random, the diffusion equations might be used as a very basic model to compare with more complex models. We can extend this simple model to more complicated and realistic movement patterns by including correlation between successive steps. The correlated random walk (CRW) is also called 'persistent' as the successive steps are correlated. In a CRW model each step tends to point in the same direction as the previous one, however the persistence fades away gradually in time and the directions become uniformly distributed in the long term [3]. We can say that a CRW model is a RW with an introduced local bias.

The discrete-time correlated random walk (CRW) [4] - [6] and its extensions [7] [8] are the foundation of the movement data models. Discrete-time models are very useful, since many methods used in the time series literature [9] can be borrowed. Also, McClintock et al. [10] suggest that the discrete-time formulations in terms of step-length and turning angle per unit time are intuitive to interpret, thus the existence of variety of discrete-time formulations and applications. Moreover, since the movement data of individual cells or animals are typically in the form of a time-series, i.e. we track the individual cell or animal at a specific location at a certain time, the discrete-time random walks are well-suited to the analysis of such data. We implement the models using computers, therefore we still need to discretize at some pre-specfied time. This time-step (the time between observations) ideally should be as small as possible, but this may not be possible due to experimental constraints or efficiency considerations. When using a CRW the persistence fades away gradually in time, therefore, a large time-step will lead to a loss in correlation, transforming the path into a simple random walk. However, some disadvantages of the discretetime models are that the computational efficiency needs to be improved and that the inference is not time scale-invariant [10]. Problems are caused by the fact that the discrete-time models often do not have a joint-model representation and require iterative simulations and thus the implementation is slow [7] [8]. One main issue of the discrete-time models is the specification of the unknown time-step length that must be done in advance [6] [11]. This causes problems dealing with irregular observations, as the choice of sampling rate might be based on considerations other than important behavioural events. These considerations might be logistical such as GPS-collar battery life [12]. Also, missing data can occur stochastically due to the devices that are used to track the animals as well as external reasons such as weather and terrain [7]. In this paper, following Turchin (1998) 
[5] and Morales et al. (2004) [7] we fit statistical models for components of discrete-time random walks. Those components include the step-length and the associated observed turning angle relative to the previous step between each pair of successive observations. The distributions we use are the Weibull distribution for the step-lengths, respectively the Wrapped Cauchy distribution for the turning angles [7]. In this paper we simulate data from a CRW model and we assume the discretization step for the original data to be $t=1$. We change the discretization step by interpolating with different time-steps and the data after interpolation resembles a real dataset. Afterwards, we fit a CRW movement model, perform inference using MCMC methods, namely the Metropolis-Hastings algorithm, and then finally perform model checking using multiple test statistics. The main aim of the paper is to assess whether the different test statistics capture the lack of model fit when fitting a discrete-time model with a different time step than the original data.

\section{Data}

We simulate data for the step-lengths from a Weibull distribution with the shape parameter 5 and the scale parameter 2. The associated observed turning angles data are simulated from a Wrapped Cauchy distribution with shape parameter 0.9. The sample size is 1000 . The time step for the original dataset is set to 1 arbitrary time unit, meaning that every time unit an independent draw takes place. We denote this dataset dataset 0 . Using cubic interpolation we sample data with a new time step. We denote this dataset dataset 1 which now resembles a real dataset. We analyse different cases when the new time steps are $0.1,0.2,0.3$, respectively 2 time units. For example, when the time step is 0.1 time units this means that we draw an observation every 0.1 time units, compared to the previous case when we draw an observation every 1 time unit. From the $\mathrm{x}$-coordinates and $\mathrm{y}$-coordinates we obtain from the interpolation we calculate the step-lengths and the associated turning angles.

\section{Models}

We let $r_{t}$ represent the observed step-length and let $\theta_{t}$ represent the associated observed turning angle at time t.

The CRW model considered is

$$
\begin{aligned}
r_{t} & \sim \text { Weibull }(\mathrm{a}, \mathrm{b}) \\
\theta_{t} & \sim \operatorname{WrapCauchy}\left(\theta_{t-1}, c\right)
\end{aligned}
$$

The Wrapped Cauchy distribution is more peaked and has heavier tails compared to other circular distributions such as the von Mises or the Wrapped Normal distribution.

The probability density function of the Weibull distribution is

$$
f(x ; a, b)=\frac{a}{b}\left(\frac{x}{b}\right)^{a-1} \exp \left(-\left(\frac{x}{b}\right)^{a}\right)
$$

where $x \geq 0, a$ is the shape parameter and $b$ is the scale parameter, both positive parameters.

The probability density function of the Wrapped Cauchy distribution is

$$
p(\theta ; c)=\frac{1-c^{2}}{2 \pi\left(1+c^{2}-2 c \cos \theta\right)}
$$

where $0 \leq \theta \leq 2 \pi$ and $c$ is the shape parameter, with $0<c<1$.

\subsection{Prior distributions}

We choose a non-informative uniform prior for all the parameters. The equations for the prior of the CRW model are

$$
p(x)=1 \text { for where } x \in\{a, b, c\} \text { and } x \in(0,1)
$$

where $a$ is the shape parameter of the Weibull distribution, $b$ is the scale parameter of the Weibull distribution and $c$ is the shape parameter of the Wrapped Cauchy distribution. 


\section{Likelihood calculation}

Suppose that data $r_{1}, \ldots, r_{n}$ are independent and identically draws from a Weibull distribution, $a$ is the shape parameter of the Weibull distribution and $b$ is the scale parameter of the Weibull distribution. For the first model, the equation for the $\log$ likelihood for the step-lengths is

$$
l(r, a, b)=\sum_{i=1}^{n} \log \left(\frac{a}{b^{a}} \times r_{i}^{a-1} \times \exp \left(-\frac{r_{i}^{a}}{b^{a}}\right)\right)
$$

Suppose that $\theta_{1}, \ldots, \theta_{n}$ are independent and identical draws from a Wrapped Cauchy distribution. For the second model, the $\log$ likelihood equation for the turning angles $\theta$ 's, where $c$ is the shape parameter, is

$$
l(\theta, c)=\sum_{i=1}^{n} \log \left(\frac{1-c^{2}}{2 \pi\left(1+c^{2}-2 c \cos \theta_{i}\right)}\right)
$$

where $0 \leq \theta_{i} \leq 2 \pi$ and $c$, the shape parameter, with $0<c<1$.

The full log likelihood for the CRW model is given by the sum of the log likelihoods from equations 4 and 5, given that the models for the step-lengths and turning angles are independent. Therefore, the joint likelihood equation factorizes and inference of the step-lengths parameters is not influenced by the inference of the turning angles.

\section{Inference}

One of our goals in this section is to infer the shape and scale parameters of the Weibull distribution and the shape parameter of the Wrapped Cauchy distribution. In order to do that, we use two MCMC samplers, one to infer the shape and scale parameters from the Weibull distribution and the other one to infer the shape parameter from the Wrapped Cauchy distribution. The algorithm chosen to infer the parameters of interest is Metropolis-Hastings. MCMC is not the natural methodological choice in this case, as there are more efficient inference techniques that can be used, such as directly sampling from the Cauchy or Weibull distribution or slice sampling [13]. However, these approaches would not be applicable to more complex models. MCMC, on the other hand, is a generally applicable tool. It was therefore chosen as a testbed for future, more general models. The proposal distribution is a symmetric Normal distribution in both cases. The MCMC sample sizes and the burn-in phase are adjusted to ensure a potential scale reduction factor of less than 1.1. When the interpolating time-step is 0.2, 0.3 and 2, the MCMC sample sizes are 20,000 and the burn-in used is 5000 iterations for the shape and scale parameters of the Weibull distribution and 1000 iterations for the shape parameter of the Wrapped Cauchy distribution. When $t=0.1$ we use more samples for the first MCMC sampler, 30,000 and a larger burn-in 20,000 for the shape parameter, respectively 15,000 iterations for the scale parameter of the Weibull distribution in order to reach convergence. For $t=1$ the number of MCMC iterations is 50,000 and the burn-in is the same as for when the time step is $0.2,0.3$ and 2. The acceptance rates were close to $40 \%$ and the step-sizes were tuned to give an acceptance probability within the desired interval between $30 \%-60 \%$.

\section{Results of the inference}

We illustrate the results of the inference in Table 1. Analysing Table 1, we can see that the shape parameters do not change much, however as the results of the interpolation the scale parameter gets divided accordingly. For example, if the interpolation step is 0.1 , then we have in total 10 times more observations, and we can see that the scale parameter is almost 10 times less (0.202) than the original scale parameter 2. For all the step-sizes we obtain similar results. It is worth noting that some samples might get lost due to the interpolation and because we need to calculate the step-lengths and the turning angles between two consecutive positions. For $\mathrm{t}=0.1$, we plot in Figure 1 the new dataset (dataset 1 ) obtained after interpolation. 
Table 1: Table of the inference results. The true values for the shape and scale parameter for the Weibull distribution are 5, respectively

2, and for the Wrapped Cauchy distribution the true value for the shape parameter is 0.9.

\begin{tabular}{|c|c|c|c|c|}
\hline \multicolumn{5}{|c|}{ Results } \\
\hline $\begin{array}{l}\text { Interpolation time- } \\
\text { step }\end{array}$ & Sample size & $\begin{array}{l}\text { Weibull Shape pa- } \\
\text { rameter mean }\end{array}$ & $\begin{array}{l}\text { Weibull Scale pa- } \\
\text { rameter mean }\end{array}$ & $\begin{array}{l}\text { Wrapped Cauchy } \\
\text { shape parameter } \\
\text { mean }\end{array}$ \\
\hline 0.1 & 9988 & $4.48 \pm 0.022 \mathrm{std}$ & $0.202 \pm 0.0004 \mathrm{std}$ & $0.983 \pm 0.0002 \mathrm{std}$ \\
\hline 0.2 & 4993 & $4.49 \pm 0.05 \mathrm{std}$ & $0.404 \pm 0.001 \mathrm{std}$ & $0.969 \pm 0.0005 \mathrm{std}$ \\
\hline 0.3 & 3328 & $4.52 \pm 0.05 \mathrm{std}$ & $0.606 \pm 0.002 \mathrm{std}$ & $0.955 \pm 0.001 \mathrm{std}$ \\
\hline 1 & 997 & $5.16 \pm 0.13 \mathrm{std}$ & $1.99 \pm 0.013$ & $0.903 \pm 0.004 \mathrm{std}$ \\
\hline 2 & 500 & $6.04 \pm 0.21 \mathrm{std}$ & $3.82 \pm 0.029 \mathrm{std}$ & $0.82 \pm 0.01 \mathrm{std}$ \\
\hline
\end{tabular}

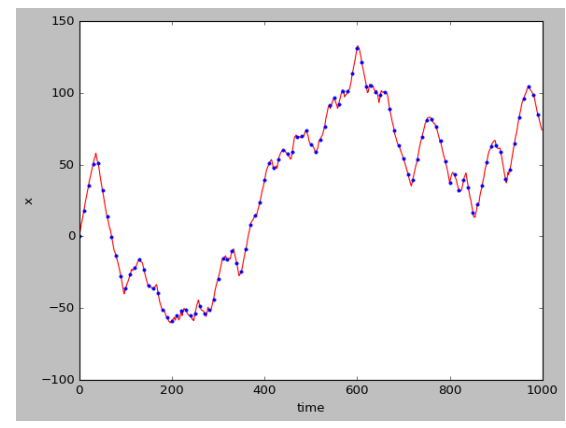

(a)

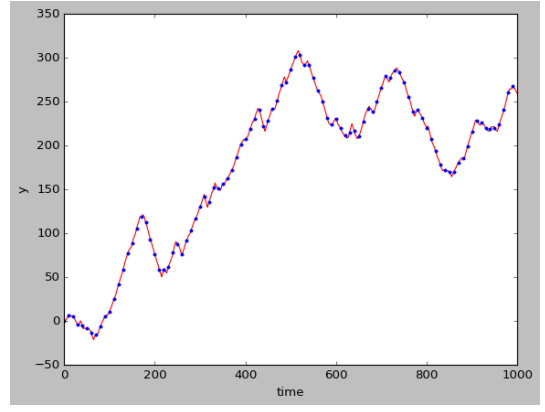

(b)

Fig. 1: Plot of the x-coordinates, respectively y-coordinates from dataset 1 (red line) and of every 5th $\mathrm{x}$-coordinate, respectively 5 th $\mathrm{y}$-coordinate from dataset 0 (blue dots).

\section{Model checking}

In this section we see whether our inferred model is a good fit to our data (dataset 1). Following Gelman et al. [14] we perform model checking: The most popular model checking approach consists of generating replicates of the sample from the posterior predictive distribution and observe the behaviour of sample summaries over repeated sampling. The goal in model checking is to calculate some statistic that allows us to measure the discrepancy between the model and the data. We calculate this statistic for the true data set and compare with the same statistic calculated in the predictive data sets. To conduct a posterior predictive check, we do the following

1. Come up with a suitable test statistic $\mathrm{T}$ that is powerful enough to detect violations of whatever assumption we are testing.

2. Calculate $\mathrm{T}$ for the observed data $\mathrm{y}: \mathrm{T}(\mathrm{y}, \phi)$, where $\phi$ is a vector of estimated parameters.

3. Calculate $\mathrm{T}$ for each draw $\mathrm{y} \_$rep from the posterior predictive distribution: this gives $\mathrm{T}$ (y_rep, $\boldsymbol{\phi} \_$rep), where $\boldsymbol{\phi} \_$rep is a vector of estimated parameters.

4. Compare the posterior predictive distribution of $\mathrm{T}$ (y_rep, $\left.\phi \_r e p\right)$ to the posterior distribution of $\mathrm{T}(\mathrm{y}, \boldsymbol{\phi})$.

5. Finally calculate the fraction of times $\mathrm{T}$ (y_rep, $\left.\phi \_r e p\right)>T(y, \phi)$. This is an estimate of the posterior predictive p-value. If our posterior predictive p-value is 'extreme' i.e. close to 0 or 1 (say 0.005 or 0.95 ), then it suggests that something in our model may be inadequate.

In our case we use two test-statistics, including the log-likelihood and the diffusion coefficient. Also, we use a visual test by plotting the average distance from the origin in dataset 1 and the average distance from the origin in the replicated datasets generated from our inferred model. 


\subsection{Log-likelihood as a test statistic}

In this section we use the log-likelihood as a test statistic to see if our inferred model is a good fit to our observed data i.e. dataset 1 , the data obtained after interpolation. The test statistic $\mathrm{T}(\mathrm{y})$ is the sum of the $\log$ likelihoods for the Weibull distribution for the step-lengths and for the Wrapped Cauchy distribution for the angle. Using the posterior means obtained from the inference in Table 1, we calculate the log likelihoods for our observed data (dataset 1). To perform model checking effectively we need at least 300 replicate datasets, however due to computational reasons we choose the number of replicate samples to be 500, as might be computationally expensive to generate more replicate datasets. We use 500 different sets of posterior samples from the two MCMC samplers (one to infer the shape and scale parameters from the Weibull distribution and the other one to infer the shape parameter from the Wrapped Cauchy distribution) to generate the replicate samples. To get to the desired number of replicate samples (500), after burn-in, we select every $10^{\text {th }}$ posterior parameter estimate and then we discard the required last number of samples. The required number of samples to discard will vary in each case depending on the MCMC sample size and the burn-in.

\subsection{Average distance from the origin}

In this section we perform model checking by using a visual test, namely the average distance from the origin plot. We calculate the average distance from the origin at each time step from 100 simulations for our observed data after interpolation (dataset 1) and for the replicate datasets obtained from the posterior samples. The sample size for the original data (dataset 0 ) is 1000. The average distance from the origin for dataset 1 is calculated using moving averages. Suppose we have a vector $\mathbf{x}=\left(x_{1}, x_{2}, \ldots, x_{n}\right)$, then for example the first two moving averages calculated are $M A_{1}=\frac{x_{1}+\cdots+x_{N}}{N}$ and $M A_{2}=\frac{x_{2}+\cdots+x_{N+1}}{N}$. In this case $n \geq N+1, n, N \in \mathbb{N}$ and $N$ is the length of a sliding window over which we wish to calculate the mean. If we do not have enough values at the end we do not calculate the average mean any further. As for the replicate samples, we generate data 100 times using the same set of parameters for each replicate sample (500 in total) to get the average distance from the origin at each time step. The first entry in the average distance vector is the average distance from the origin at time 0 , the second entry is the average distance from the origin at time step $t$, the third entry is the average distance from at time step $2 t$ etc. The time step for the original data (dataset 0 ) is $t=1$ and the time step for dataset 1 will depend on the interpolation time step. For example, let $\phi=\left(\phi_{1}, \phi_{2}, \phi_{3}\right)$ be a set of estimated parameters obtained from the inference, where $\phi_{1}, \phi_{2}$ are the shape and scale parameters for the Weibull distribution and $\phi_{3}$ is the shape parameter for the Wrapped Cauchy distribution. Using this set of parameters we generate data 100 times, calculate the distance from the origin at each time-step for every simulation, and then take the average for each entry. More precisely, suppose the distance from the origin vector for the $\mathrm{k}^{\text {th }}$ simulated sample is $\mathbf{v}^{\mathbf{k}}=\left(v_{0}^{k}, v_{t}^{k}, v_{2 t}^{k}, \ldots\right)$ where $v_{0}^{k}$ is the distance from the origin to the origin (the first entry is always going to be 0$), v_{t}^{k}$ is the distance from the origin to the position at time-step $t$ and so on, where $t$ is the interpolation time-step and $\mathrm{k}$ is a natural number between 1 and 100. Thus, the average distance for the origin across all simulations is $\mathbf{u}=\left(\frac{\sum_{k=1}^{k=100} v_{0}^{k}}{100}, \frac{\sum_{k=1}^{k=100} v_{t}^{k}}{100}, \frac{\sum_{k=1}^{k=100} v_{2 t}^{k}}{100}, \ldots\right)$. We repeat the same procedure to calculate the average distance from the origin for the remaining replicated samples. It is important to stress that each replicate sample is generated using a different set of parameters, but we average across 100 simulations using the same set of parameters.

\subsection{The diffusion coefficient as a test statistic}

In this section we use the diffusion coefficient as a test statistic. We use the following mathematical equation $u=\sqrt{2 D \tau}$ [15], where $\mathrm{u}$ is the average distance from the origin vector from the simulations at each time step, $\tau$ is the vector of time-steps and $D$ is the diffusion coefficient. Using the results from the previous section, we calculate the diffusion coefficient for datasets 1 and for the replicate samples. We get that $u^{2}=2 D \tau$, and then we can do linear regression to calculate the slope D.

\subsection{Model checking results}

In this section we show the results of the model checking using the two test statistics and the visual test. In Table 2 we show the results using log likelihood as a test statistic and in Figures 2 and 3 we plot scaled histograms of the log-likelihood test statistic for the replicate samples (T (y_rep)) and the log-likelihood test statistic for dataset 1 (T (y)). In all cases the log-likelihoods were re-scaled to adjust to the fact that the replicate sample size is 1000 , compared to the dataset 1 sample size. In Table 3 we show the results using the diffusion coefficient as a test statistic. Moreover, in Figure 4 (a) we illustrate 
the average distance from the origin for dataset 1 and for the replicate samples when $t=0.1$. In Figures 4 (b) and 4 (c) we plot histograms of the replicate samples and the test statistics $\mathrm{T}(\mathrm{y})$ (35.41, respectively 39.57) using the diffusion coefficient for the cases when $t=0.1$, respectively $t=2$. For the cases when $t$ is $0.2,0.3$ or 2 , all the plots using the average distance from the origin are very similar to Figure 4 (a). Likewise, when $t$ is 0.2 or 0.3 , the histograms using the diffusion coefficient as a test statistic are very similar to Figures 4 (b) and 4 (c).

Table 2: Model checking results using re-scaled log-likelihood per data point as a test statistic.

\begin{tabular}{|l|l|l|l|}
\hline $\mathrm{t}$ & The dataset 1 sample size & $\mathrm{T}(\mathrm{y})$ & Bayesian $\mathrm{p}$-value \\
\hline 0.1 & 9988 & 2.75 & 1 \\
\hline 0.2 & 4993 & 1.37 & 0.008 \\
\hline 0.3 & 3328 & 0.589 & 0.006 \\
\hline 1 & 1000 & -1.362 & 0.63 \\
\hline 2 & 500 & -2.45 & 0.57 \\
\hline
\end{tabular}

Table 3: Model checking results using the diffusion coefficient as a test statistic.

\begin{tabular}{|l|l|l|l|}
\hline $\mathrm{t}$ & The dataset 1 and the replicate datasets sample size & $\mathrm{T}(\mathrm{y})$ & Bayesian p-value \\
\hline 0.1 & 9988 & 35.41 & 0 \\
\hline 0.2 & 4993 & 35.28 & 0 \\
\hline 0.3 & 3328 & 35.17 & 0 \\
\hline 2 & 500 & 39.57 & 0.002 \\
\hline
\end{tabular}

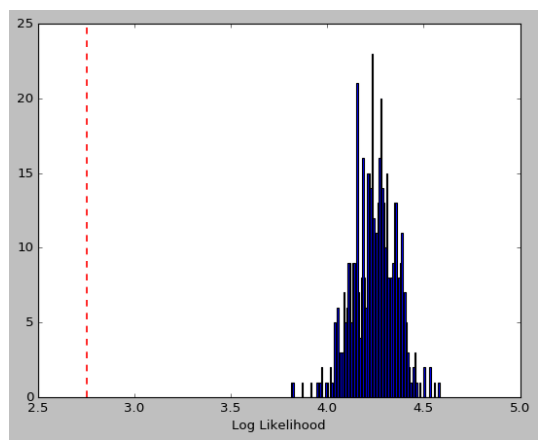

(a) Scaled histogram of $\mathrm{T}\left(\mathrm{y} \_\right.$rep) and $\mathrm{T}(\mathrm{y})$ (dashed red line) for $\mathrm{t}=0.1$.

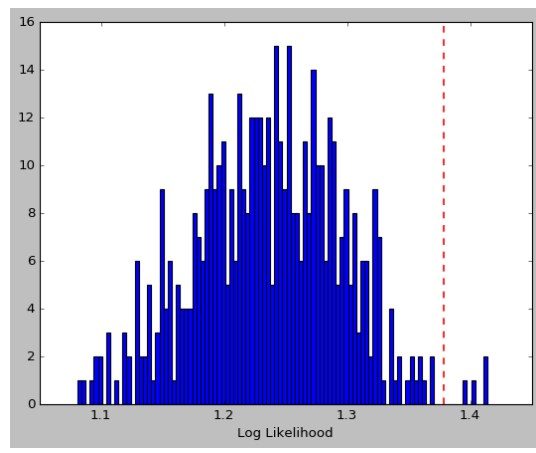

(b) Scaled histogram of $\mathrm{T}\left(\mathrm{y} \_\right.$rep $)$and $\mathrm{T}(\mathrm{y})$ (red dashed line) for $\mathrm{t}=0.2$.

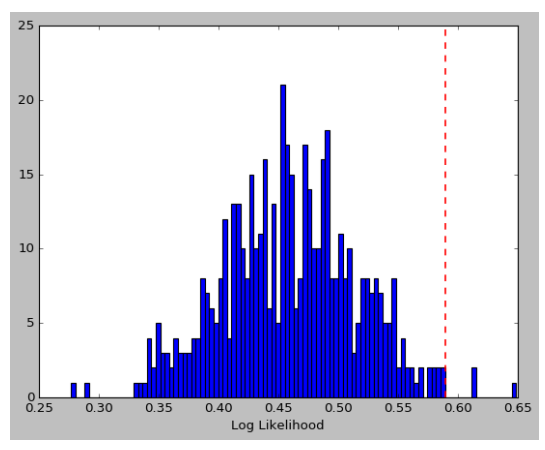

(c) Scaled histogram of $\mathrm{T}\left(\mathrm{y} \_\right.$rep $)$and $\mathrm{T}(\mathrm{y})$ (red dashed line) for $\mathrm{t}=0.3$.

Fig. 2: Model checking using log likelihood as a test statistic. 


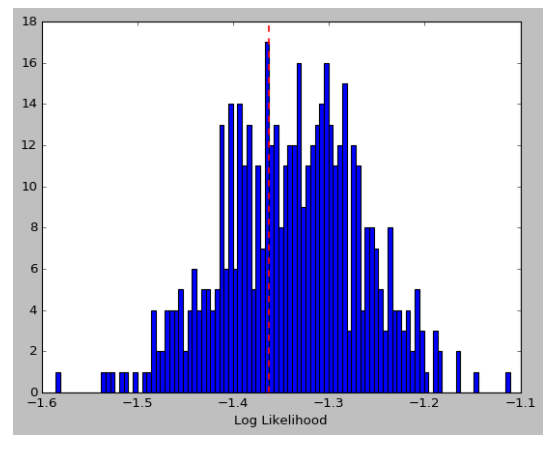

(a) Scaled histogram of T(y_rep) and $\mathrm{T}(\mathrm{y})$ (red dashed line) for $\mathrm{t}=1$.

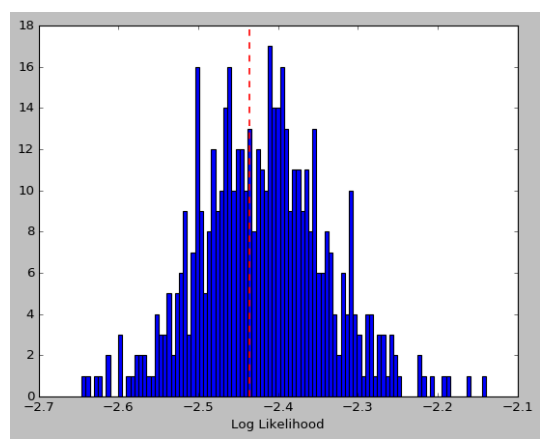

(b) Histogram of $\mathrm{T}\left(\mathrm{y} \_\right.$rep) and $\mathrm{T}(\mathrm{y})$ (red dashed line) for $\mathrm{t}=2$.

Fig. 3: Model checking using log likelihood as a test statistic.

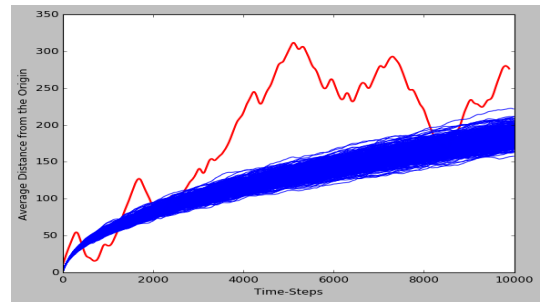

(a) Average distance from the origin plots for $\mathrm{t}=0.1$. The red line represents the average distance from the origin for dataset 1 , and the blue lines represent the average distance from the origin for the replicate samples.

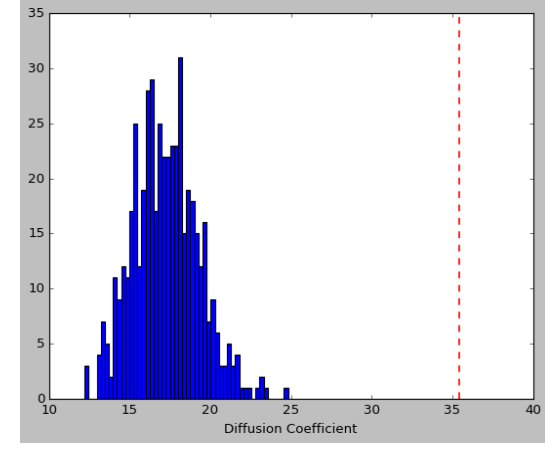

(b) Histogram of $\mathrm{T}\left(\mathrm{y} \_\right.$rep) and $\mathrm{T}(\mathrm{y})$ (red dashed line) for $\mathrm{t}=0.1$.

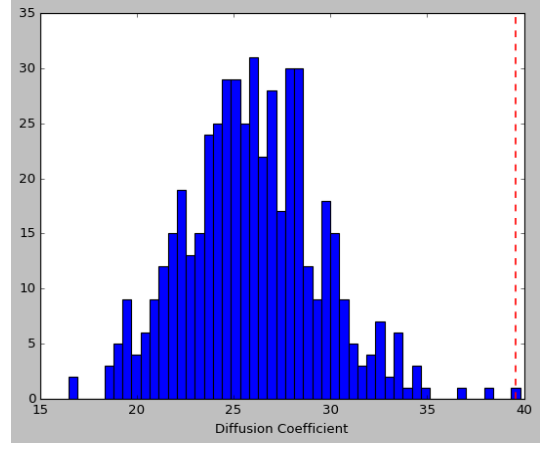

(c) Histogram of $\mathrm{T}\left(\mathrm{y} \_\right.$rep) and $\mathrm{T}(\mathrm{y})$ (red dashed line) for $\mathrm{t}=2$

Fig. 4: Model checking using an average distance from the origin plot and a diffusion coefficient test statistic.

In almost all of the cases we found out that there is a model mismatch between our inferred model and the observed data (dataset 1) illustrated in Table 2 (extreme Bayesian p-values), Figures 2 and 3 (using log-likelihood), Table 3 (extreme Bayesian p-values) and Figures 4 (a), 4 (b) and 4 (c) (using the average distance from the origin plot and the diffusion coefficient as a test statistic). The only exception occurred when $\mathrm{t}=2$ when using the log-likelihood as test-statistic, illustrated in Table 2 (Bayesian p-value is 0.57) and in Figure 3 (b). Also, when we fit a model with the same-time step as the original data ( $\mathrm{t}=$ 1) we get that there is no evidence of a model mismatch illustrated in Table 2 (Bayesian p-value is 0.63) and Figure 3 (a), as expected.

\section{Conclusions}

Mathematical modelling of animal movement movement is becoming ever more important in quantitative ecology, and with the improvement in GPS tagging technologies, increasing amounts of data are rapidly becoming available. This opens the door to statistical inference, to address the related challenges of parameter estimation, hypothesis testing and uncertainty quantification. In the present paper, we have focused on a model that is the centrepiece of the animal movement literature: a Markovian discrete time random walk model. While very basic, this model is an essential building block from which many more advanced models are constructed by model extension. We have set up a Bayesian inference scheme based on sampling parameters from the posterior distribution. In order to be generalisable to more complex and advanced models, we have applied a general-purpose Markov chain Monte Carlo (MCMC) sampler. This addresses the challenges of parameter estimation (e.g. posterior means) and uncertainty quantification (credible intervals). To address the challenge of hypothesis testing, we have generated synthetic data with a mismatch in the sampling frequency, to test procedures for detecting a 
systematic mismatch between the model and the data. In our study, we have evaluated a Bayesian approach to model critique based on summary statistics and posterior predictive p-values. The idea is to select an informative summary statistic and compute it from the original data. We then compare this value with the posterior distribution of the same summary statistic based on the model. This posterior distribution is obtained by drawing a sample of parameters from the posterior distribution, simulating data for each sampled parameter, and then computing the summary statistic for each simulated data set. The posterior predictive p-value quantifies how far in the tails the summary statistic of the true data lies, with values close to 0 or 1 indicating a systematic model mismatch. Any choice of summary statistic incurs an inevitable information loss, though, and our study has shown that the log likelihood is not sufficiently informative for consistent mismatch indication. The diffusion coefficient, on the other hand, has consistently indicated any model mismatch related to wrong sampling rates, and has thus turned out to be a more reliable model mismatch indicator. Besides testing Bayesian model critique procedures, our study has highlighted a fundamental shortcoming of discrete-time movement models, namely the need to select a sampling time interval (inverse sampling frequency) in advance. In our future work, we will apply Bayesian inference techniques to continuous-time movement models, like Gaussian processes, which are more flexible and inherently avoid this limitation.

\section{References}

[1] G. P. Panotopoulos, S. Aguayo and Z. S. Haidar, "Nonmotile Single-Cell Migration as a Random Walk in Nonuniformity: The "Extreme Dumping Limit" for Cell-to-Cell Communications," Journal of Healthcare Engineering, vol. 2018, Article ID 9680713, 2018.

[2] E.A. Codling, N.A. Hill, J.W. Pitchford and S.D Simpson, "Random walk models for the movement and recruitment of reef fish larvae," Mar Ecol Prog Ser, vol. 279, pp. 215-224, 2004.

[3] S. Benhamou, "Detecting an orientation component in animal paths when the preferred direction is individual dependent," Ecology, vol. 87, pp. $518-528,2006$.

[4] P.M. Kareiva, N. Shigesada, "Analyzing insect movement as a correlated random walk," Oecologia, vol. 56, pp. 234-238, 1983.

[5] P. Turchin, Quantitative analysis of movement: measuring and modeling population redistribution in animals and plants. Sunderland, Massachusetts: Sinauer Associates, 1998.

[6] P. Bovet, S. Benhamou, "Spatial analysis of animals' movements using a correlated random walk model," J. Theor. Biol., vol. 131, pp. 419-433, 1988.

[7] J. Morales, D. Haydon, J. Friar, K. Holsinger, and J. Fryxell, "Extracting more out of relocation data: Building movement models as mixtures of random walks," Ecology, vol. 85, pp. 2436-2445, 2004.

[8] B. McClintock, R. King, L. Thomas, J. Matthiopoulos, B. McConnell, and J. Morales, "A general discrete-time modeling framework for animal movement using multistate random walks," Ecological Monographs, vol. 82, pp. 335-349, 2012.

[9] R. Anderson-Sprecher, J. Ledolter, "State-Space Analysis of Wildlife Telemetry Data," Journal of The American Statistical Association - J AMER STATIST ASSN., vol. 86, pp. 596-602, 1991.

[10] B.T. McClintock, D.S. Johnson, M.B. Hooten, J. Hoef and J. M Morales, "When to be discrete: the importance of time formulation in understanding animal movement," Movement Ecology, vol. 2, no. 21, 2014.

[11] C.H. Fleming, J.M. Calabrese, T. Mueller, K.A. Olson, P. Leimgruber, and W.F. Fagan, "From fine-scale foraging to home ranges: A semi-variance approach to identifying movement modes across spatio-temporal scales," The American Naturalist, vol. 183, no. 5, pp. E154-67, 2014.

[12] C.H. Fleming, J.M. Calabrese, T. Mueller, K.A. Olson, P. Leimgruber, and W.F. Fagan, "Non-Markovian maximum likelihood estimation of autocorrelated movement processes," Methods in Ecology and Evolution, vol. 5, pp. 462-472, 2014.

[13] K. P. Murphy, Machine Learning: A Probabilistic Perspective (Adaptive Computation and Machine Learning Series). Cambridge, Massachusetts; London, England: The MIT Press, 2012.

[14] A. Gelman, J. Carlin, H. Stern, D. Dunson, A. Vehtari, and D. Rubi, Bayesian Data Analysis. New York: CRC Press, 2013.

[15] E.A. Codling, M.J. Plank and S. Benhamou, "Random walk models in biology," J R Soc Interface, vol. 5, no. 25, pp. 813-834, 2008. 